\title{
Impact of Macroeconomic Variables on Stock Market Indices Value: A Cross-Country Indices Study
}

Rohini Mariappan*, Nikita Hari† and Amalendu Jyotishi ${ }^{\ddagger}$

\section{Abstract}

Complete unpredictability and the contagion effect of stock markets could pose significant challenges for the entire financial markets of the world. Moreover, it is an incontrovertible truth that the variations in stock market indices is an integral part of the dynamics of economic activity and can propel social moods and expectations. In fact, the stock market has predicted 10 out of the last 3 recessions. In this context, this paper examines the present scenario of the stock markets across the under-developed, developing and developed economies of the globe. With the aid of various literature and article reviews, the variables that affect the stock market movement are determined to be inflation, industrial production index, foreign institutional investments, exchange rates, bank rates, unemployment rate, credit rating of various countries and the country's current state of development. As the stock market follows a non linear trend, this paper employs an auto-regression model to arrive at the degree of influence of each variable on the stock market index change. Ultimately the paper aids the policy makers and the investors to focus on the most influencing factor to

\footnotetext{
* Students of MBA-MS Program Amrita School of Business and University at Buffalo (State University of New York) (ASBUB), Kasavanahalli, Carmelaram P:O:, Off Sarjapur Road, Bangalore - 560 035; mrohini19@gmail.com.

† (ASBUB), nikigang@gmail.com.

‡ Associate Professor (ASBUB), amalendu.jyotishi@gmail.com.
} 
end in better stock picking decisions thereby leading to superior earning capability.

Keywords: Stock market indices, Financial markets, Autoregression model, Various economies

JEL Classification: G15, E44

\section{Introduction}

As the saying goes, the stock market is the barometer of business; the stock market indices reflect the economic status of many countries. It influences the investors' confidence, capital accumulation, international trading, industrial production, liquidity management and henceforth the entire economical development. Most likely, the investment opportunities and the performance of the financial markets in general are impelled by the macro-economic scenario across the globe. Ultimately, it is important to promptly arrive at a decision of which macroeconomic variable affects the stock market to a larger degree so as to get a deeper insight on stock picking decisions that aid the end investors, the normal people like us who are the driving forces of any economy.

The paper captures the world's best, moderate and the worst performing stock markets in terms of stock market indices change and the macro-economic variables (inflation, industrial production index, foreign institutional investments, exchange rates, bank rates, credit rating of various countries and the country's current state of development) that affect the stock market indices.

\section{Review of Literature}

The Indian stock market index is taken as a function of exchange rates against USD, Index of Industrial Production (IIP), and Wholesale Price Index (WPI) as according to Dharmendra (2009). Chen et al. (1986) is of the opinion that changes in stock returns are inclusive of the increase in the industrial production, variation in the risk premium and the yield curve, fluctuations in inflation rates, etc. In order to affirm on the relationship between stock 
market indices and the other factors, Dharmendra (2009) carried out unit root test and Granger causality test. This paper takes into account time series data. The results of the correlation matrix show that IIP and SENSEX have a strong correlation. Since causality and correlation are two different entities whose results are not the same; the causality tests show that only IIP influences the SENSEX and that the other two variables are not responsible for causing fluctuations in the stock market index.

Anthony Kyereboah-Coleman and Kwame F. Agyire-Tettey, (2008) in their journal on the movement of Ghana stock exchange indices with respect to various macro-economic variables indicate that the lending rates could primarily contribute to the performance of the stock market. The two models - Neo classical monetary growth model and Keynesian models put forth contradictory remarks on the interest rates' impact on the investment, income and savings thereafter. Rate of inflation, exchange rate, fiscal position, GDP, growth rate etc were other variables that could impact the stock market. The results showed that high lending rates had a negative impact on the business. Inflation also had its share of effect on the stock market indices but not too evident because the present inflation level hardly has an impact on the market. The only disadvantage to this paper was that the effect of macro-economic variables on the stock market index was studied only for one country.

Shiu-Sheng Chen(2003) in his journal on prediction of bear stock market identified the following variables to have an impact on the stock market index : interest rate spreads, inflation rates, aggregate output, unemployment rate, federal fund rates, federal government debts, nominal exchange rates. The aim of his paper was to help policy makers given the predictability of movement in the stock market indices thereby helping them to form market-timing strategies. The paper contributed to 2 types of approachesparametric and non-parametric. Furthermore, a predictive regression model was made use of to test the hypothesis on the $\beta$ value.

Tantatape and Komain (2007) through their study on the variations in the stock market indices of Thailand attributed to money supply, 
industrial production index, exchange rate and oil prices observed that the money supply had a positive impact on the stock market. This was proved through the Granger's causality test. Furthermore, the impact was seen to a greater extent in the pre-financial crisis than the post-financial crisis in Thailand.

According to Aima, Hira and Zaheer (2011), the increasing interest rates and exchange rates caused the returns to decline. Whereas inflation showed a positive impact on the stock market. The two major methodologies adopted was co-integration test and vector auto regression which was used to test the long term and short time relationship between the macroeconomic variables and its impact on the stock market indices.

David, Mark and Jesper (2005), in their study, included macroeconomic factors such as interest rates, industrial production, term spread and unemployment rate and money stock. The impact of these on the stock market was tested over 12 industrialised countries and was observed that the interest rates are strong predictors and better reliable to estimate the returns on stock.

Findings from Seyed, Zamri, Yew Wah (2011) show the relationship between the stock market indices and 4 major macroeconomic variables such as crude oil price, money market, inflation and industrial production in China and India. The effect of industrial production and inflation seems to be positive in India whereas the effect of crude oil prices and money supply is negative in India. Unit test and Multi-variate co-integration test were used to determine the relationship between the two variables.

According to T. Sampath (2011), the Auto- Regressive Distributed Lag (ARDL) approach was used to test co-integration. The effect of Wholesale Price Index (WPI), real effective rate and Index of Industrial Production (IIP) seemed to show stronger effect on the stock prices. Exchange rates and Inflation seemed to show a negative relationship to the stock prices.

The following were the possible variables that were obtained from the review of literature - IIP, Money supply, Exchange rate, Interest rate spreads, Inflation rates, Crude Oil Prices, Lending rates, Real 
effective exchange rate, Wholesale price index and Index of Industrial Production.

Econometric Model and Sample size estimation:

The econometric model used is linear in nature.

Where,

$$
\begin{aligned}
& Y=f(X 1, X 2, X 3, X 4, X 5, X 6, X 7) \\
& \text { implies } Y=\alpha+\beta 1 X 1+\beta 2 X 2+\beta 3 X 3+\beta 4 X 4+\beta 5 X 5+\beta 6 X 6 \\
& +\beta 7 X 7+U i
\end{aligned}
$$

Y-Stock market index value change (2009-10)

X1- Interest Rate Spread

X2- Internet Users/100

X3- GDP growth (2009-10)

X4- Foreign Direct Investments

X5- Money Supply Growth

X6- Stock Turnover

X7- Real and Effective Exchange rate index

Out of the 214 countries, only 37 of them are listed in the world federation of exchanges and 44 are non-listed. The sample size consists of 24 countries that constitute a combination of developed and developing economies in the ratio 1:2 the data obtained consists of information over the year 2010. This constitutes about $65 \%$ of the population (i.e.) 24 out of 37 countries.

\section{Methodology}

A linear regression model was run on SPSS over the obtained data to check on the stability and the significance of the independent variables

\section{Data}

The data sources available in the IMF website, World Bank website, the websites of the various stock markets all over the world and various other journal articles serve as our database. This paper reflects a set of cross sectional data of the annual stock market indices change and the annual macro-economic variables across 24 countries. 
Of the possible variables listed through literature review, the following variables were considered: Interest Rate spread, FDI, GDP Growth, Money Supply Growth, and Real and Effective Exchange Rate index. Apart from these 5 variables were introduced 2 new variables: Internet Users and Stock Turnover.

Table: 1

\begin{tabular}{|c|c|c|c|c|c|c|c|c|}
\hline 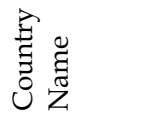 & 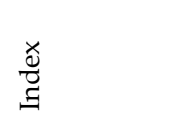 & 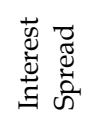 & $\begin{array}{l}\vec{\Xi} \\
\stackrel{\Xi}{\Xi} \\
\stackrel{\Xi}{\Xi}\end{array}$ & $\vec{\rho}$ & $\hat{0}$ & 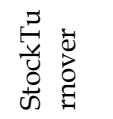 & 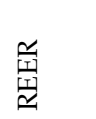 & 芯 \\
\hline Australia & AORD & 3.067 & 775.89 & 6.059 & 2.257 & 90.077 & 115.18 & 9.376 \\
\hline Bahrain & & 6.023 & 55 & -1.808 & 4.5 & 1.539 & 89.45 & 10.509 \\
\hline Bulgaria & SOFIX & 7.068 & 45.98 & 1.3609 & 0.4 & 2.754 & 121.05 & 6.303 \\
\hline Chile & IPSA, IGPA & 3.004 & 45 & 6.3509 & 6.095 & 19.712 & 108.45 & 10.561 \\
\hline China & SSE & 3.06 & 34.39 & 1.2511 & 10.4 & 164.372 & 118.66 & 18.948 \\
\hline Colombia & IGBC & 5.72 & 36.5 & 3.3808 & 4.001 & 13.44 & 120.32 & 11.458 \\
\hline C. Republic & PX Index & 4.806 & 68.64 & 4.9209 & 2.739 & 29.417 & 122.45 & 1.876 \\
\hline Hungary & BUX & 2.669 & 65.16 & 3.9109 & 1.258 & 94.528 & 106.12 & 4.2 \\
\hline Israel & TA-100 & 2.93 & 65.39 & -2.8009 & 4.846 & 66.66 & 115.39 & -11.004 \\
\hline Japan & Nikkei 225 & 1.098 & 77.64 & -5.9010 & 4.435 & 114.488 & 102.68 & 1.919 \\
\hline Malaysia & KLSE & 2.521 & 56.3 & -4.309 & 7.194 & 27.067 & 108.81 & 7.348 \\
\hline Mexico & IPC & 4.074 & 31.05 & 6.6409 & 5.519 & 27.306 & 92.72 & 12.767 \\
\hline Netherlands & AEX Index & -0.597 & 90.71 & -6.6010 & 1.69 & 98.372 & 99.02 & 4.028 \\
\hline N. Zealand & NZX 50 & 1.672 & 83.01 & 1.2808 & 1.9 & 34.724 & 94.94 & 8.359 \\
\hline Nigeria & NSXA & 11.064 & 28.43 & 5.139 & 7.824 & 12.538 & 117.93 & 9.31 \\
\hline Pakistan & KSE 100 & 5.898 & 16.78 & 1.9709 & 4.144 & 36.181 & 103.39 & 15.053 \\
\hline Philippines & PSE Index & 4.453 & 25 & 6.8208 & 7.632 & 22.552 & 126.8 & 10.897 \\
\hline Romania & BET-10 & 6.759 & 40.02 & 2.9609 & 0.948 & 5.428 & 104.19 & 6.817 \\
\hline Russia & RTS Index & 4.808 & 43.31 & -9.2009 & 4.3 & 85.714 & 125.93 & 24.59 \\
\hline Singapore & FTSE Group & 5.174 & 71.14 & 1.8910 & 14.763 & 82.879 & 111.28 & 8.611 \\
\hline S. Africa & JSE & 3.368 & 12.33 & 1.3909 & 2.89 & 39.599 & 101.23 & 6.934 \\
\hline Switzerland & Swiss Market & 2.653 & 82.17 & -4.4010 & 2.714 & 75.602 & 107.5 & 5.501 \\
\hline Ukraine & PFTS index & 5.313 & 44.59 & 5.7609 & 4.1 & 7.477 & 99.07 & 22.691 \\
\hline Zambia & & 13.516 & 10.13 & 6.348 & 7.61 & 9.161 & 125.99 & 29.859 \\
\hline
\end{tabular}

\section{Tests and Interpretations}

Best Fit of the Line: The estimates of the model were obtained from the unstandardised beta co-efficient got through the linear regression model. The error terms were calculated by taking the difference between $Y$ (dependent variable) actual and estimate. The sum of all the error terms obtained was zero. This shows that the model has a best fit line. 
The Chow test was also done to show structural stability in the model and hence the model proved no structural break in the model.

Table: 2

\begin{tabular}{|c|c|c|c|c|}
\hline \multirow[t]{2}{*}{$\begin{array}{l}\text { Country } \\
\text { Name }\end{array}$} & \multicolumn{2}{|c|}{$\begin{array}{c}\text { Stock market Indices- (S\&P Global } \\
\text { Equity } \% \text { change) }\end{array}$} & \multirow{2}{*}{$\begin{array}{l}\text { Estimate } \\
\mathrm{y}-\end{array}$} & \multirow[t]{2}{*}{ Error } \\
\hline & Indices & $\mathrm{y}$ & & \\
\hline Australia & $\begin{array}{l}\text { All Ordinaries, } \\
\text { S\&P/ASX } 200\end{array}$ & 12.49092 & -3.35154 & 15.84245661 \\
\hline Bahrain & & 9.96791 & 11.49532 & -1.52741197 \\
\hline Bulgaria & SOFIX & -15.1638 & 3.09932 & -18.263168 \\
\hline Chile & IPSA, IGPA & 47.24033 & 43.48376 & 3.756569958 \\
\hline China & $\begin{array}{l}\text { SSE Composite } \\
\text { Index }\end{array}$ & 6.914683 & 8.520687 & -1.60600381 \\
\hline Colombia & $\begin{array}{l}\text { IGBC, COL20, } \\
\text { COLCAP }\end{array}$ & 44.05827 & 30.211 & 13.84727059 \\
\hline $\begin{array}{l}\text { Czech } \\
\text { Republic }\end{array}$ & PX Index & 0.20595 & 5.489962 & -5.28401215 \\
\hline Hungary & BUX & -10.7507 & -10.9733 & 0.222675024 \\
\hline Israel & $\begin{array}{l}\text { TA-100 Index (Tel } \\
\text { Aviv 100) }\end{array}$ & 7.362251 & -0.2376 & 7.599847542 \\
\hline Japan & Nikkei 225 & 9.614292 & 6.388457 & 3.225835603 \\
\hline Malaysia & KLSE & 35.06396 & 42.90104 & -7.83708227 \\
\hline Mexico & IPC Index & 26.59595 & 31.34764 & -4.75169543 \\
\hline Netherlands & AEX Index & 1.152789 & 9.977841 & -8.82505216 \\
\hline $\begin{array}{l}\text { New } \\
\text { Zealand }\end{array}$ & NZX 50 Index & 5.230025 & 10.79224 & -5.56221919 \\
\hline Nigeria & $\begin{array}{l}\text { NSXA-B } \\
\text { index (The All- } \\
\text { Share Index)[6] }\end{array}$ & 20.29219 & 6.980786 & 13.31140105 \\
\hline Pakistan & KSE 100 Index & 15.32022 & 24.43258 & -9.11235466 \\
\hline Philippines & PSE Index & 56.66901 & 55.65219 & 1.016823516 \\
\hline Romania & BET-10 & -6.58282 & 0.660122 & -7.24293902 \\
\hline $\begin{array}{l}\text { Russian } \\
\text { Federation }\end{array}$ & RTS Index (RTSI) & 21.67312 & 28.45272 & -6.77959643 \\
\hline Singapore & $\begin{array}{l}\text { FTSE } \\
\text { Group indices }\end{array}$ & 18.43888 & 27.08247 & -8.64358557 \\
\hline $\begin{array}{l}\text { South } \\
\text { Africa }\end{array}$ & JSE & 32.0867 & 28.49477 & 3.591929357 \\
\hline Switzerland & Swiss Market Index & 11.03874 & 4.258032 & 6.780712541 \\
\hline Ukraine & PFTS index & 53.84306 & 32.49824 & 21.34481854 \\
\hline Zambia & & 17.38908 & 22.49429 & -5.10520831 \\
\hline
\end{tabular}


Multicollinearity was used to check if there is any correlation between the independent variables. The factors checked are Tolerance and VIF. Both are inversely related and any VIF rating below 8 is considered to not have problems of multicollinearity.

It was also observed that all the seven variables were proved to be statistically significant with $90 \%$ confidence interval.

Table: 3

\begin{tabular}{|l|l|l|l|l|l|}
\hline Model 1 & Coefficients & $\begin{array}{l}\text { Standard } \\
\text { Error }\end{array}$ & t-Value & Sig. & VIF \\
\hline (constant) & 3.323 & 27.888 & & 0.119 & \\
\hline Interest Rate spread & -6.786 & 1.371 & -1.056 & -4.949 & 3.060 \\
\hline Internet Users & -0.366 & 0.155 & -0.444 & -2.359 & 2.381 \\
\hline GDP growth & 3.627 & 0.858 & 0.616 & 4.228 & 1.424 \\
\hline Stock turnover & -0.331 & 0.083 & -0.736 & -3.987 & 2.29 \\
\hline Money supply & 0.998 & 0.367 & 0.429 & 2.721 & 1.665 \\
\hline FDI & $-1.643 \mathrm{e}-10$ & 0.000 & -0.285 & -1.830 & 1.627 \\
\hline Exchange Rate & 0.479 & 0.258 & 0.272 & 1.857 & 1.441 \\
\hline
\end{tabular}

Heteroscadasticity : The distribution of the square of the error terms with respect to the y-estimates was found to show no significant pattern and hence the distribution was said to be homoscadastic in nature.

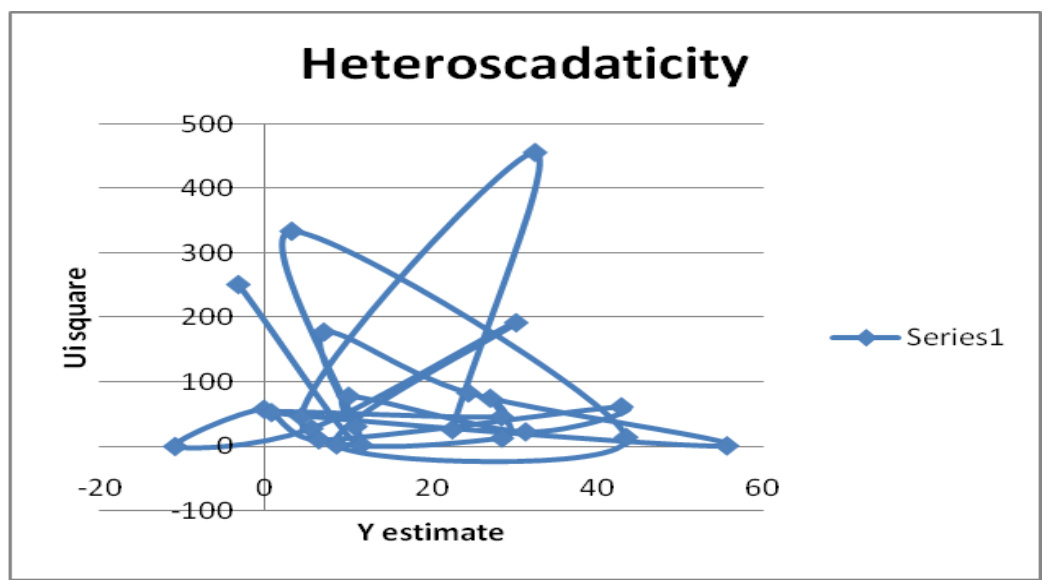

Fig:1 
Table: 3

\begin{tabular}{|l|c|l|}
\hline Country name & square & \multicolumn{1}{|c|}{ Y estimate } \\
\hline Australia & 250.9834 & -3.35154 \\
\hline Bahrain & 2.332987 & 11.49532 \\
\hline Bulgaria & 333.5433 & 3.09932 \\
\hline Chile & 14.11182 & 43.48376 \\
\hline China & 2.579248 & 8.520687 \\
\hline Colombia & 191.7469 & 30.211 \\
\hline Czech Republic & 27.92078 & 5.489962 \\
\hline Hungary & 0.049584 & -10.9733 \\
\hline Israel & 57.75768 & -0.2376 \\
\hline Japan & 10.40602 & 6.388457 \\
\hline Malaysia & 61.41986 & 42.90104 \\
\hline Mexico & 22.57861 & 31.34764 \\
\hline Netherlands & 77.88155 & 9.977841 \\
\hline New Zealand & 30.93828 & 10.79224 \\
\hline Nigeria & 177.1934 & 6.980786 \\
\hline Pakistan & 83.03501 & 24.43258 \\
\hline Philippines & 1.03393 & 55.65219 \\
\hline Romania & 52.46017 & 0.660122 \\
\hline RussianFederation & 45.96293 & 28.45272 \\
\hline Singapore & 74.71157 & 27.08247 \\
\hline South Africa & 12.90196 & 28.45272 \\
\hline Ukraine & 455.6013 & 32.49824 \\
\hline Zambia & 26.06315 & 22.49429 \\
\hline
\end{tabular}

Auto-correlation - the correlation between the error terms were found not to exist. This was supported by the data on Durbin Watson co-efficient which was obtained as 1.976. Correlation between the independent variables were found to be moderate in nature (i.e.) between 0.4 and 0.6 majorly.

Table: 4

\begin{tabular}{|c|c|c|c|c|c|c|}
\hline \multicolumn{7}{|c|}{ Coefficient Correlation } \\
\hline Variables & REER & $\begin{array}{c}\text { Stock } \\
\text { Turn-Over }\end{array}$ & $\begin{array}{l}\text { Money } \\
\text { Supply }\end{array}$ & $\begin{array}{c}\text { GDP } \\
\text { Growth }\end{array}$ & FDI & $\begin{array}{c}\text { Internet } \\
\text { Users }\end{array}$ \\
\hline REER & 1.000 & & & & & \\
\hline Stock turnover & -0.343 & 1.000 & & & & \\
\hline Money Supply & 0.144 & -0.232 & 1.000 & & & \\
\hline GDP Growth & -0.021 & -0.246 & -0.050 & 1.000 & & \\
\hline FDI & -0.043 & -0.259 & -0.045 & -0.294 & 1.000 & \\
\hline Internet User & 0.066 & -0.312 & 0.328 & 0.005 & 0.370 & 1.000 \\
\hline
\end{tabular}




\section{Ranking}

The ranking was done by calculating the technical efficiency which is the ratio of $y$-actual to $y$ - frontier. The S\&P rating assumes a different base year (1941-43) as compared to our ranking which is on the basis of just the values obtained during the year 2010.

The ones is yellow signify round about the same ranking whereas those highlighted in green show drastic difference in ranking.

Table: 5

\begin{tabular}{|c|c|c|c|c|}
\hline $\mathbf{Y}^{*}$ & $\mathrm{Y} / \mathrm{Y}^{*}$ & \multirow{2}{*}{\multicolumn{3}{|c|}{$\begin{array}{c}\text { My Ranking } \\
\text { S\&P }\end{array}$}} \\
\hline Y Frontier & $\begin{array}{l}\text { Technical } \\
\text { efficiency }\end{array}$ & & & \\
\hline 53.84272 & 1.000006 & 1 & Ukraine & 1 \\
\hline 51.55548 & 0.85458 & 4 & Chile & 2 \\
\hline 76.99667 & 0.735993 & 3 & Philippines & 3 \\
\hline 64.82824 & 0.7287 & 2 & Colombia & 4 \\
\hline 28.32527 & 0.716399 & 5 & Nigeria & 5 \\
\hline 17.99294 & 0.694212 & 8 & Malaysia & 6 \\
\hline 49.83925 & 0.643804 & 22 & Romania & 7 \\
\hline 64.24552 & 0.545781 & 21 & Czech Republic & 8 \\
\hline 52.69213 & 0.504742 & 24 & Hungary & 9 \\
\hline 49.7972 & 0.435228 & 16 & Pakistan & 10 \\
\hline 25.60251 & 0.431159 & 9 & Mexico & 11 \\
\hline 43.83877 & 0.39666 & 10 & Russian Federation & 12 \\
\hline 48.42695 & 0.380757 & 13 & Singapore & 13 \\
\hline 21.10689 & 0.348808 & 7 & South Africa & 14 \\
\hline 27.73294 & 0.346674 & 14 & Israel & 15 \\
\hline 45.77706 & 0.33467 & 20 & Netherland & 16 \\
\hline 32.8398 & 0.303531 & 11 & Switzerland & 17 \\
\hline 29.86517 & 0.23153 & 17 & Bahrain & 18 \\
\hline 32.13673 & 0.162743 & 19 & New Zealand & 19 \\
\hline 31.32232 & 0.036804 & 6 & Australia & 20 \\
\hline 26.83444 & 0.007675 & 23 & Bulgaria & 21 \\
\hline 22.0046 & -0.29916 & 15 & Japan & 22 \\
\hline 24.4438 & -0.62036 & 18 & China & 23 \\
\hline 10.37115 & -1.03659 & 12 & Zambia & 24 \\
\hline
\end{tabular}




\section{Findings and Recommendations}

\begin{tabular}{|l|l|l|}
\hline $\begin{array}{l}\text { Sl. } \\
\text { No }\end{array}$ & Findings & Recommendations \\
\hline 1 & $\begin{array}{l}\text { High inverse variation between } \\
\text { interest rate spread and Stock } \\
\text { market index (-1.1) }\end{array}$ & $\begin{array}{l}\text { Spread must keep varying according to } \\
\text { the economy (Lending rate to increase, } \\
\text { Deposit rate to decrease) }\end{array}$ \\
\hline 2 & $\begin{array}{l}\text { Internet users per 100, FDI are } \\
\text { inversely related to the } \\
\text { dependent variable }\end{array}$ & $\begin{array}{l}\text { Exchange traded regulations must be } \\
\text { tightened, FIIs are important for stocks } \\
\text { trading } \rightarrow \text { Policy implications }\end{array}$ \\
\hline 3 & $\begin{array}{l}\text { Developing economies occupy } \\
\text { the first five positions }\end{array}$ & $\begin{array}{l}\text { Contagion effect policies can be } \\
\text { implemented in such developing } \\
\text { countries }\end{array}$ \\
\hline 4 & Ranking differences & $\begin{array}{l}\text { S\&P's base year is 1941-43 for stock } \\
\text { market performance and the inclusive } \\
\text { criteria is in higher limits }\end{array}$ \\
\hline
\end{tabular}

\section{Summary of tests and Interpretations}

\begin{tabular}{|c|c|c|c|c|}
\hline Test & $\begin{array}{c}\text { Significance of the } \\
\text { test }\end{array}$ & Result & Interpretation & $\begin{array}{l}\text { Is the } \\
\text { model } \\
\text { stable }\end{array}$ \\
\hline Sum(Ui) & Best fit line & 0 & Best Fit (BLUE) & Yes \\
\hline Chow test & Structural Stability & $\mathrm{F}=0.435$ & $\begin{array}{l}\text { No structural } \\
\text { Break }\end{array}$ & Yes \\
\hline Histogram & $\begin{array}{l}\text { Error } \\
\text { termdistribution }\end{array}$ & Normal curve & $\begin{array}{l}\text { Normally } \\
\text { distributed Ui }\end{array}$ & Yes \\
\hline $\begin{array}{l}\text { Multi- } \\
\text { collinearity }\end{array}$ & $\begin{array}{l}\text { Correlation among } \\
\text { Independent } \\
\text { Variables }\end{array}$ & $\begin{array}{l}\text { VIF }<10 \text {, No great } \\
\text { fluctuation, } \\
\text { Tolerance } \\
<1 \text {, Rsqr }\end{array}$ & $\begin{array}{l}\text { Independent } \\
\text { variables are } \\
\text { not co }\end{array}$ & Yes \\
\hline $\begin{array}{l}\text { Heteroscadas } \\
\text { ticity }\end{array}$ & $\begin{array}{l}\text { Distribution of } \\
\text { Uisqr }\end{array}$ & $\begin{array}{l}\text { No pattern between } \\
\text { Uisqr and } \\
\text { Y estimate }\end{array}$ & $\begin{array}{l}\text { Homoscadastic } \\
\text { distribution }\end{array}$ & Yes \\
\hline $\begin{array}{l}\text { Auto-Co- } \\
\text { relation }\end{array}$ & $\begin{array}{l}\text { Correlation between } \\
\text { error terms }\end{array}$ & $\begin{array}{l}\text { Durbin Watson Co- } \\
\text { efficient }=1.976\end{array}$ & $\begin{array}{l}\text { Co-relation bet } \\
\mathrm{Ui}, \mathrm{Uj}=0\end{array}$ & Yes \\
\hline F-statistic & $\begin{array}{l}\text { Sample regression } \\
\text { prediciting } \\
\text { Population }\end{array}$ & $\begin{array}{l}\mathrm{F}=0.001 \text { at } 90 \% \\
\text { confidence }\end{array}$ & $\begin{array}{l}\text { Adequate } \\
\text { model }\end{array}$ & Yes \\
\hline T-statistic & Ivs significance & $\begin{array}{l}\text { All } 7 \text { Ivs' } t<0.1 \text { at } \\
90 \% \text { confidence, But } \\
\text { insignifican }\end{array}$ & Significant Ivs & Yes \\
\hline R sqr & $\begin{array}{l}\text { Variation in all } \\
\text { Ivs affecting DV }\end{array}$ & $\begin{array}{l}\text { Rsqr }=0.762 \\
\text { Adjusted } \\
\text { Rsqr }=0.657\end{array}$ & $\begin{array}{l}\text { Optimal } \\
\text { Variations (No } \\
\text { over/under) }\end{array}$ & Yes \\
\hline $\begin{array}{l}\text { Co-efficient } \\
\text { Co-relation }\end{array}$ & $\begin{array}{l}\text { Co-relation } \\
\text { among Ivs }\end{array}$ & $\begin{array}{l}\text { All values are } \\
\text { moderate }<=0.6,>=- \\
0.4\end{array}$ & $\begin{array}{l}\text { Not highly } \\
\text { Co-related }\end{array}$ & Yes \\
\hline
\end{tabular}




\section{Limitations of the study}

- Stability in growth cannot be observed

- Non-linear pattern of stock market makes forecasting difficult

\section{Scopes for improvement}

- Ranking methodology can be improved

- Extension of model to panel data of the last decade

\section{References}

Brahmasrene, T., \& Jiranyakul, K. (2007). Co-integration and causality between stock index and macroeconomic variables in an emergin market. Academy of Accounting and Financial Studies Journal .

Chen, N.F., Roll, R., Ross, A., \& Stephen. (1986). Economic forces and the stock market. Journal of Business , 383-403.

Chen, S. S. (2009). Predicting the bear stock market: Macroeconomic variables as leading $1=$ indicators. Journal of Banking and Finance, 211223.

Hosseini M.S., Ahmad, Z., \& Weh-Lai, Y. (2011). Role of macroeconomice variables on stock market index in China and India. International Journal of Economics and Finance.

Khan, A., Ahmad, H., \& Abbas, Z. (2011). Impact of macroeconomic factors on stock prices. Interdisciplinary Journal of Contemporary Reserch in Business , 3 (1).

Kyereboah, C.A., \& Agyire, T.K. F. (2008). Impact of the macroeconomic indicators on stock market performance: The case of the Ghana Stock Exchange. The Journal of Risk Finance, 365-378.

Rapach E, D., Wohar E, M., \& Rangvid, J. (2005). Macrovariables and international stock return predictability. International Journal of Forecasting , 137-166.

T, S. (2011). Macroeconomic variables and stock prices in India : An emperical analysis. The IUP Journal of Monetary Economics . 